\title{
Influenza Virus-like Particles Containing HA, NA, and M1 Induced Protection in Chickens against a Lethal Challenge with the Highly Pathogenic H5N1 Avian Influenza Virus
}

Motofumi Shimizu', Satomi Yanase ${ }^{1}$, Chang Myint OO$^{2}$, Masatoshi Okamatsu ${ }^{3}$, Yoshihiro Sakoda ${ }^{3}$, Hiroshi Kida ${ }^{3}$ and Hiroshi Takaku ${ }^{1,2 \star}$

${ }^{1}$ Department of Life and Environmental Sciences, Chiba Institute of Technology, 2-17-1 Tsudanuam, Narashino, Chiba 275-0016, Japan

${ }^{2}$ High Technology Research Center, Chiba Institute of Technology, 2-17-1 Tsudanuam, Narashino, Chiba 275-0016, Japan

${ }^{3}$ Laboratory of Microbiology, Graduate School of Veterinary Medicine, Hokkaido University, Sapporo 060-0818, Japan

\begin{abstract}
Background: Intermittent outbreaks of highly pathogenic avian influenza virus (flu) infections illustrate the potential for pandemic spread of this deadly disease, thus making the development of sufficient supplies of safe vaccines a necessity. Influenza virus-like particles (VIps) have been suggested as a promising non-egg or nonmammalian cell culture-based candidate for vaccination against flu. VIps containing hemagglutinin have previously been shown to promote protection against homologous viral strains. In this report, we describe the development of an H5N1 flu VIp vaccine involving only three flu viral structural proteins, (i.e., HA, NA, and M1), which were derived from an avian flu A/duck/Hokkaido/vac-1/2004 (H5N1) virus. The H5N1 VIps produced from insect cells exhibited hemagglutination and neuraminidase activities and generated an immune response in BALB/c mice. We additionally performed viral challenge studies using chickens.
\end{abstract}

Methodology and results: Vlps consisting of the hemagglutinin (HA), neuraminidase (NA), and matrix 1 (M1) proteins of A/duck/Hokkaido/vac-1/2004 (H5N1) were transferred using baculovirus within Spodoptera frugiperda (Sf9) cells. Mice were first immunized with Vlps, and the immune response was compared between animals vaccinated with HA and NA-HA-negative M1 VIps. The IgG levels of HA-M1 VIp- and NA-M1 VIp-treated groups were observed, and 5-fold higher levels of $\mathrm{H} 5 \mathrm{~N} 1$-specific antibodies were induced in the groups of mice immunized with HA-NA-M1 VIp. The HA-NA and HA-NA-M1 VIps vaccines induced IgG2a and IgG2b antibodies as well as IgG1 antibodies, indicating that both Th1 and Th2 immune responses were induced. Furthermore, NA-M1 immunization induced $\lg G$ and $\lg G 1$ isotype antibodies and led to low levels of IgG2a and IgG2b. Additionally, all chickens immunized with HA-NA-M1 VIp were protected against deadly infections with the highly pathogenic avian flu virus $\mathrm{A} /$ chicken/Yamaguchi/4/2004 (H5N1).

Conclusions: Intramuscular administration of $\mathrm{H} 5$ VIps conferred immunity against a deadly viral challenge. The H5 Vlp vaccine was more successful at raising Th1-biased protective responses, including IgG2a production. Thus, flu Vlps offer an important approach for immunization, especially if a pandemic occurs. Recognizing the current state of vaccination strategies, it is imperative to investigate the associated immunogenicity and defensive capabilities of $\mathrm{H} 5 \mathrm{Vlps}$ in comparison with inactivated whole virus and attenuated live H5N1 viruses. These results support the continued development of VIps as a formulation for a vaccine against flu infection.

Keywords: Influenza virus; H5N1; Virus-like particle; Hemagglutinin (HA); Neuraminidase (NA); Protection; Mouse; Chicken

\section{Introduction}

Human infection with the highly pathogenic avian influenza A H5N1 remains an indisputable and remarkably complex global health challenge despite the current focus on the pandemic outbreaks initiated by the rise of influenza A H1N1/09 virus [1-3]. The highest incidence of human infections caused by H5N1 occurred in Hong Kong in 1997 with eighteen cases and six deaths [4,5], and the virus has continued to be associated with a high case-fatality rate [3]. Recently, human infection has only occurred through close contact with animals (mainly poultry) infected with $\mathrm{H} 5 \mathrm{~N} 1$ virus. $\mathrm{H} 5 \mathrm{~N} 1$ infection does not currently satisfy the third pandemic criterion, sustained human-tohuman transmission, despite reports of transmission between humans $[6,7]$. Vaccine development has been a key component of pandemic preparedness plans [8-11]. Vaccination is an essential countermeasure to combat seasonal and pandemic flu. Flu vaccines have been prepared as live, inactivated whole virus generated in embryonated hens' eggs, using a strategy developed more than 60 years ago, or as split virions. Recently, mammalian cells have been utilized to produce flu vaccines in Europe. The major immunogens in these vaccines are the viral proteins hemagglutinin (HA) and neuraminidase (NA). Although production of these vaccines is feasible, it is also inefficient with respect to time and manufacturing constraints. Therefore, when a flu pandemic arises, it takes nearly 4-6 months after the identification of the virus to prepare the first distributable quantities of vaccine. Early studies with inactivated split virion vaccines that comprise $\mathrm{H} 5 \mathrm{~N} 1$ strains demonstrated that such vaccines could be safely administered to human subjects [12-15]. In fact, many countries wish to assemble a stockpile of $\mathrm{H} 5 \mathrm{~N} 1$ vaccine. However, H5N1 vaccine stockpiles will be severely limited by the absence of sufficient $\mathrm{H} 5$ vaccine antigen because of limited worldwide

*Corresponding author: Hiroshi Takaku, Department of Life and Environmental Sciences and High Technology Research Center, Chiba Institute of Technology, 2-17-1 Tsudanuma, Narashino, Chiba 275-0016, Japan, E-mail: hiroshi.takaku@it-chiba.ac.jp

Received August 20, 2013; Accepted August 27, 2013; Published August 30, 2013

Citation: Shimizu M, Yanase S, Myint COO, Okamatsu M, Sakoda Y, et al. (2013) Influenza Virus-like Particles Containing HA, NA, and M1 Induced Protection in Chickens against a Lethal Challenge with the Highly Pathogenic H5N1 Avian Influenza Virus. J Vaccines Vaccin 4: 201. doi: 10.4172/2157-7560.1000201

Copyright: ( 2013 Shimizu M, et al. This is an open-access article distributed under the terms of the Creative Commons Attribution License, which permits unrestricted use, distribution, and reproduction in any medium, provided the original author and source are credited. 
Citation: Shimizu M, Yanase S, Myint COO, Okamatsu M, Sakoda Y, et al. (2013) Influenza Virus-like Particles Containing HA, NA, and M1 Induced Protection in Chickens against a Lethal Challenge with the Highly Pathogenic H5N1 Avian Influenza Virus. J Vaccines Vaccin 4: 201. doi: $10.4172 / 2157-7560.1000201$

Page 2 of 7

production. Recently, a few noteworthy strategies have been used to increase antigen production. One promising engineering technique uses recombinant noninfectious virus-like particles (Vlps) that present structurally native, immunologically relevant viral antigens. Various studies have demonstrated the behavior, immunogenicity, and defensive capability of flu Vlps. These Vlps comprised the HA, NA, and matrix 1 (M1) proteins, which are processed by the synchronous expression of these genes in an insect cell baculovirus expression system [16-23]. We have previously shown that intranasal immunization with a recombinant baculovirus containing the flu virion HA protein protects mice against a lethal challenge with influenza $A$ and $B$ viruses [24]. In this report, we describe the development of an H5N1 flu Vlp vaccine that contained three flu infection structural proteins, HA, NA, and M1, derived from the avian flu A/duck/Hokkaido/vac-1/2004 (H5N1) virion [25]. The H5N1 Vlps derived from insect cells showed hemagglutination and neuraminidase activities and generated immune responses in $\mathrm{BALB} / \mathrm{c}$ mice. We similarly performed experimental studies utilizing chickens.

\section{Materials and Methods}

\section{Cloning of HA, NA, and M1 genes}

The cDNA fragments containing the influenza HA, NA, and M1 genes of avian influenza A/duck/Hokkaido/Vac-1/2004 (H5N1) were cloned into the pCR2.1-TOPO vector. PCR was performed on pCR2.1-TOPO-M1, pCR2.1-TOPO-NA, and pCR2.1-TOPO-HA, using the KOD-Plus system (TOYOBO, Osaka, Japan), with genespecific oligonucleotide primers. The following primer pairs were used to synthesize the HA, NA, and M1 fragments, respectively: 5'- HA S6SmaI (5'-cgcccgggaccATGGAGAaaATAGTACTTCTTTTTG-3') and HA A6SmaI (5'-cgcccggggtTTAAATGCAAATTCTGCATTG-3'); NA S3BglII (5'-gaagatctgccaccATgAATCCAATCCAAATC AAAAGATAATAAC-3') and NA A2XbaI (5'-gctctagagCATCGTCAACATCCACAGA-3'); and M1 S2XbaI (gctctagagccgccaccATGAGTCTTCTAACCGAGGTC) and M1 A2Xba I(gctctagagCATCGTCAACATCCACAGA). After RT-PCR, cDNA fragments encoding the flu $\mathrm{HA}, \mathrm{NA}$, and $\mathrm{M} 1$ genes were cloned into the pMOS Blue vector (GE Healthcare Bio-Science Co, London, UK). The nucleotide sequences of the HA, NA, and M1 genes of pMOS-Blu-HA, $\mathrm{NA}, \mathrm{M} 1$ were determined by DNA sequencing and were discovered to be indistinguishable from the encoded alignments.

\section{Generation of recombinant baculoviruses}

The M1 gene was cloned into the pAcDB3 bacmid exchange vector (BD Biosciences) (which had previously been processed with $\mathrm{XbaI}$ ) downstream of the polyhedrin promoter, as an XbaI DNA fragment (1.0 $\mathrm{kb})$. The resultant baculovirus exchange plasmid containing influenza genes was designated pAcDB3-M1. The HA and M1 genes were cloned into a bacmid exchange vector (SmaI-processed AcDB3-M1 plasmid DNA) downstream of the p10 promoter, as SmaI DNA fragments (1.8 $\mathrm{kb}$ ) (Figure 1). The NA gene was then cloned into the same vector (after EcoRI-BglII processing of the $\mathrm{pAcDB} 3-\mathrm{M} 1$ plasmid DNA) downstream of the p10 promoter, as EcoRI-BglII DNA pieces (1.4 kb) (Figure 1). The two resultant baculovirus exchange plasmids containing the flu virus genes were designated pAcDB3-HA-M1 and pAcDB3-NA-M1.

Finally, a bacmid exchange vector that allowed the expression of each of the three flu genes was constructed, with the genes cloned into the SmaI-processed pAcDB3-NA-M1 plasmid DNA downstream of the p10 promoter, as $S m a I$ DNA sections $(1.8 \mathrm{~kb})$ (Figure 1). This process resulted in a plasmid, pAcDB3-HA-NA-M1, that encoded the HA, $\mathrm{NA}$, and M1 genes, each within its own expression cassette. The vector also incorporated a p10 and polyhedrin promoters and translation termination signal. The DNA fragment from pNVAX1250 containing the NA, M1, and HA expression cassettes was then transferred into bacmids, and Spodoptera frugiperda (Sf9) insect cells (ATCC CRL-1711) were transfected with the bacmids to generate recombinant baculovirus [17]. Titers of the recombinant baculovirus stocks were determined by agarose plaque tests in Sf9 cells. Recombinant baculoviruses were utilized to infect Sf9 cells. Vlps were harvested at $72 \mathrm{~h}$ posttransfection. Recombinant Vlps were purified by a $20-60 \%(\mathrm{w} / \mathrm{v})$ discontinuous sucrose density gradient and sedimented by ultracentrifugation for 16 $\mathrm{h}$ at $27,000 \mathrm{rpm}$ and $4^{\circ} \mathrm{C}$. Fractions $(1 \mathrm{ml})$ were collected from the top of each gradient using a 1-ml syringe. Expression of HA, NA and M1 proteins in the purified Vlp preparation was confirmed by Western blot analysis, as described by Pushko et al. [17]. Hemagglutination titration assays were used to measure HA content, as described in the general procedure by Donald and Isaacs [26]. The purified Vlps were stored under refrigeration $\left(4-8^{\circ} \mathrm{C}\right)$.

\section{Hemagglutinin (HA) and hemagglutination inhibition (HI)} tests

Two-fold serial dilutions of flu Vlps in PBS were prepared and
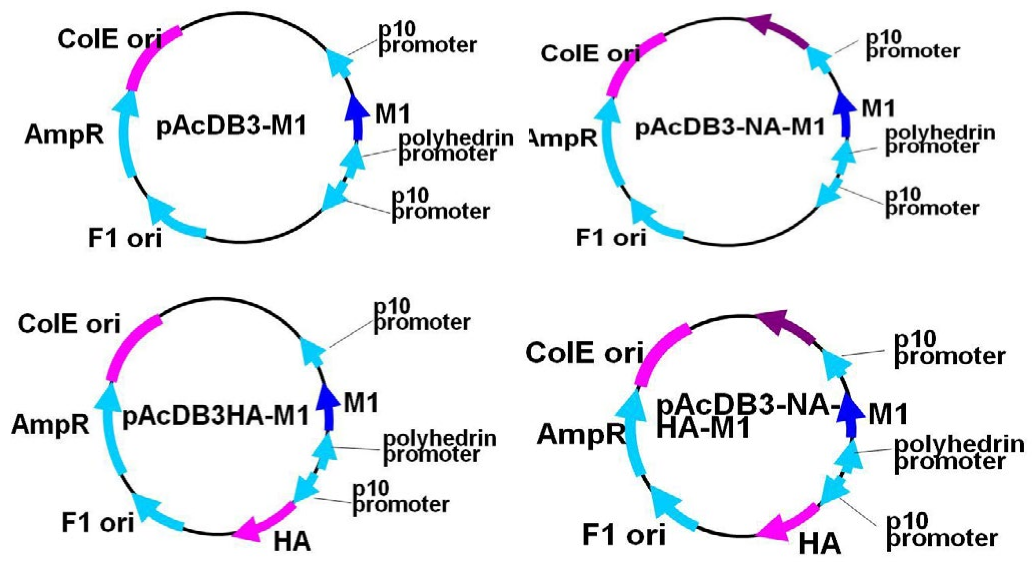

Figure 1: (A) Constructs for the individual expression or co-expression of influenza proteins. The polyhedrin promoter (Polh), p10 promoter, and avian influenza A/duck/ Hokkaido/Vac-1/2004 (H5N1) genes are indicated: HA, hemagglutinin; NA, neuraminidase; and M1, matrix protein. 
Citation: Shimizu M, Yanase S, Myint COO, Okamatsu M, Sakoda Y, et al. (2013) Influenza Virus-like Particles Containing HA, NA, and M1 Induced Protection in Chickens against a Lethal Challenge with the Highly Pathogenic H5N1 Avian Influenza Virus. J Vaccines Vaccin 4: 201. doi: $10.4172 / 2157-7560.1000201$

Page 3 of 7

incubated at $25^{\circ} \mathrm{C}$ for $2 \mathrm{~h}$ with $0.5 \%(\mathrm{v} / \mathrm{v})$ chicken red blood cells. The degree of hemagglutination was assessed, and the best dilution for agglutinating $0.5 \%(\mathrm{v} / \mathrm{v})$ chicken red blood cells was determined [26]. An HI test was completed according to previously described methods [27], utilizing four hemagglutination units of $\beta$-propiolactoneinactivated avian flu A/duck/Hokkaido/vac-1/2004 (H5N1) combined with serial dilutions of antibodies from Vlp-vaccinated BALB/c mice and then assessing the agglutination of chick red blood cells.

\section{Serologic test for the determination of antibody responses}

Female BALB/c mice (6 weeks old) were purchased from Nippon SLC (Hamamatsu, Japan) and housed under humane conditions consistent with the guidelines and regulations of our institutional committee. Mice (7 per group) were intramuscularly immunized with $10 \mu \mathrm{g}$ of Vlps in $50 \mu \mathrm{l}$ of PBS twice at 3-week intervals (weeks 0 and 3). Blood samples were collected retro-orbitally prior to vaccination and at various time points after the final immunization (weeks 1 and 4). The blood samples were allowed to clot prior to centrifugation, then serum was isolated and stored at $-20^{\circ} \mathrm{C}$ until titration.

Peripheral blood samples were collected before and after immunization at various time points. The $\mathrm{H} 5 \mathrm{~N} 1$ virion-specific antibody titers were determined by ELISA, as previously described [19]. Briefly, an inactivated flu $\mathrm{H} 5 \mathrm{~N} 1$ virion was used as a coating antigen on 96-well microtiter plates (Nunc, Thermo Fisher Scientific, MA, USA), which were then incubated overnight at $4^{\circ} \mathrm{C}$. The wells were then washed three times with PBS containing 0.05\% Tween 20 (PBST) and blocked with PBST containing $3 \% \mathrm{BSA}$ for $1 \mathrm{~h}$ at $37^{\circ} \mathrm{C}$. Serially diluted serum samples were included and incubated for $2 \mathrm{~h}$ at $37^{\circ} \mathrm{C}$. Finally, HRP-conjugated anti-rodent goat IgG (GE Healthcare, London, UK), IgG1 (Southern Biotechnology Associates, AL, US), IgG2a (Invitrogen, California, US) and IgG2b (Invitrogen) were utilized as secondary antibodies. The ELISA endpoint titers were determined as the most notable dilutions that yielded optical densities greater than the mean optical density plus 3 standard deviations of analogously diluted negative control sera. To confirm the antibody titers, absorbance was read at $450 \mathrm{~nm}$.

\section{Animals and experimental design}

The vaccine was prepared by emulsifying $250 \mu \mathrm{g}$ of the Vlp antigen product with Freund's Complete Adjuvant (FCA, BD Difco, Michigan, USA) at a ratio of 30:70 (v/v). To assess the vaccine effectiveness, chickens were challenged intranasally with a highly pathogenic avian flu virus, A/chicken/Yamaguchi/7/2004 (H5N1) [28]. Four-week-old White Leghorn SPF chickens were divided into two groups (3 per group). Chickens were immunized three times at 2-week intervals (weeks 0, 2, and 4) with $250 \mu \mathrm{g}$ of Vlp antigen plus adjuvant via intramuscular injections. As a non-immunized control group, 3 additional White Leghorn chickens were injected with an emulsion of distilled water in FCA in the same proportion as the Vlp antigen. Three weeks after the final round of immunization, under Animal Biosafety Level 3 conditions, the chickens were challenged intranasally with the 1,000 CLD50 of A/chicken/Yamaguchi/7/2004 (H5N1). To confirm the reproducibility of the challenge at day 2 post-challenge (p.c.), intraoral and cloacal swabs were gathered from each chicken, as previously described [13]. The clinical data were computed as the mean score for each fowl for each observation. Blood specimens were gathered at 0,2 , 4,6 , and 7 weeks post-vaccination. Intraoral and cloacal swabs were performed on day 2 p.c. to assess viral shedding. Animal experiments were approved by the Institutional Animal Care and Use Committee of the Graduate School of Veterinary Medicine, Hokkaido University (endorsement number: 10-0007), and all experiments were performed in accordance with the guidelines of this committee.

\section{Results}

\section{Production of the Vlp vaccine for A/duck/Hokkaido/Vac- 1/2004 (H5N1)}

Recombinant baculoviruses were generated for individual expression of HA, NA, or M1 proteins as well as for the co-expression of these proteins (Figure 1). Influenza H5N1 Vlps were produced in insect cells infected with one of four recombinant baculoviruses, expressing HA-NA-M1, HA-M1, NA-M1, or M1 of 220 A/duck/Hokkaido/Vac1/2004 (H5N1) virus. Purified H5N1 Vlps contained HA-NA (Figure 2A), HA (data not shown), and NA (data not shown). as well as M1, as indicated by Western blot analysis, as described by Pushko et al. [17]
A

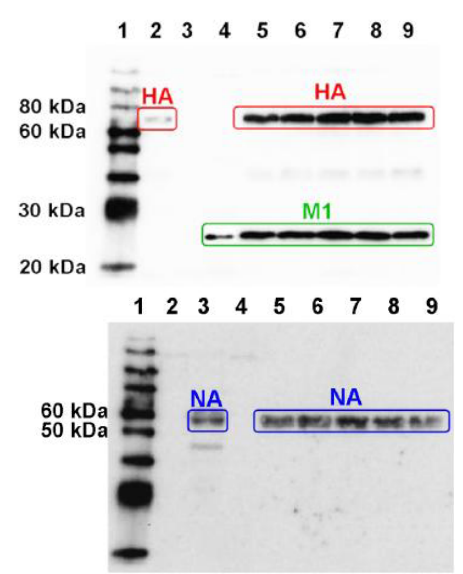

B

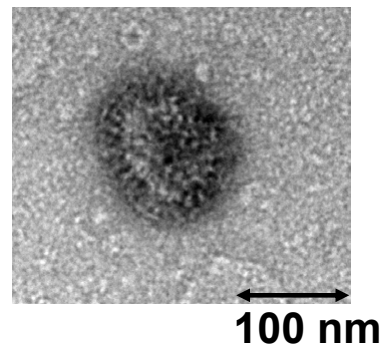

Figure 2: (A) Analysis of purified HA-NA-M1 Vlp by Western blotting using chicken serum DK/Hok/Vac-1/04 specific for H5N1 (upper panel) and rabbit monoclonal anti-neuraminidase antibody (bottom panel). Lane 1: Marker; Lane 2: Positive control, HA; Lane 3: Positive control, NA; Lane 4: Positive control, M1. Lane 5: HA-NA-M1 VLP. (B) Negative staining electron microscopy of H5N1 influenza VIps comprising the HA, NA, and M1 proteins. Bars represent 100 nm. 
Citation: Shimizu M, Yanase S, Myint COO, Okamatsu M, Sakoda Y, et al. (2013) Influenza Virus-like Particles Containing HA, NA, and M1 Induced Protection in Chickens against a Lethal Challenge with the Highly Pathogenic H5N1 Avian Influenza Virus. J Vaccines Vaccin 4: 201. doi: $10.4172 / 2157-7560.1000201$

Page 4 of 7

(Figure 2). The HA titers of HA-NA-M1 Vlps and HA-M1 Vlps were $6.8 \times 108 \mathrm{HA}$ and $3.4 \times 108 \mathrm{HA}$, respectively. Additionally, the size and morphology of influenza Vlps were examined by electron microscopy (Figure 2B). The morphology of Vlps resembled that of influenza virus particles with spikes on their surfaces, which are characteristic of flu HA proteins on virions. The particle sizes ranged from approximately 80 to $120 \mathrm{~nm}$. Taken together, these results demonstrate that Sf9 cells infected with rBVs expressing HA, NA and M1 generated particles resembling influenza virions in terms of morphology and size.

\section{The immune response elicited by Vlp vaccination}

To determine whether influenza Vlps induce immune responses specific to flu HA, mice were intramuscularly immunized with 10 $\mu \mathrm{g}$ of flu Vlps containing HA-M1, NA-M1, HA-NA-M1 or an HAnegative Vlp containing M1 alone (referred to as HA-M1 Vlp, HANA-M1 Vlp, and M1 Vlp, respectively) at 3-week intervals. The serum immune response stimulated after two rounds of immunization was assessed at various time points (weeks 0,4 , and 8). Sera diluted 100 to 1000 -fold was utilized to verify the A/duck/Hokkaido/vac-1/2004 (H5N1)-specific total IgG by ELISA. At week 8 post-vaccination, the total IgG levels of groups treated with HA-M1-Vlp or NA-M1 Vlp were observed, and 5-fold higher levels of H5N1-specific antibodies were detected in HA-NA-M1 Vlp-immunized mice (Figure 3A). At weeks 4 and 8 post-immunization, the levels of antibodies were unaltered. No significant levels of antibodies specific for A/duck/Hokkaido/vac$1 / 2004$ (H5N1) were detected in the groups of mice vaccinated with HA or HA-NA-negative M1 Vlps. Limited data are available on the protective role of neuraminidase (NA), the second major glycoprotein. A subsequent study reported that flu Vlps containing M1 and NA are capable of inducing immunity to homologous as well as antigenically dissimilar flu A strains [29]. The mice immunized with NA Vlps were $100 \%$ protected against lethal infections by the homologous A/ $\mathrm{pr} / 8 / 1934$ (H1N1) and heterosubtypic A/Philippines/1982 (H3N2) infections. In this study, antibodies were analyzed in HA-M1-Vlpimmunized groups.

To further characterize the immune responses, we assessed the pattern of isotypes induced by immunization with Vlps. IgG1, which is indicative of a T helper type 2 (Th2)-biased response, was discovered to be the prevailing antibody subclass raised in the vaccinated groups at week 8 post-immunization (Figure 3B). Moreover, HA-M1 and HANA-M1 Vlps groups induced IgG2a and IgG2b antibodies at week 8 post-immunization, showing Th1-biased responses (Figure 3C). The HA-M1 and HA-NA-M1 Vlp vaccines raised IgG2a and IgG2b antibodies as well as the IgG1 subclass, indicating that both Th1 and Th2 immune responses were elicited (Figures 3B and 3C). However, higher levels of $\mathrm{H} 5 \mathrm{~N} 1$-specific antibodies were induced in HANA-M1 Vlp-immunized mice than in HA-M1 Vlp-vaccinated mice. Finally, considerable levels of total IgG and IgG1 isotype antibodies, but low levels of IgG2a and IgG2b antibodies, were observed after immunization with NA-M1 Vlps (Figure 3C).

\section{Immunization with HA-NA-M1 Vlps protects chickens against an $\mathrm{H} 5 \mathrm{~N} 1$ challenge}

To evaluate the immunogenicity of HA-NA-M1 Vlps, fourweek-old White Leghorn SPF chickens were placed into two groups. Chickens were intramuscularly vaccinated three times (weeks 0,2 , and 4) at 2-week intervals with $250 \mu \mathrm{g}$ of Vlp vaccine with adjuvant. At 2, 4, 6, and 7 weeks after priming, we determined the serum levels of HI titers (Table 1). The serum HI antibody titers to the immunization strain were 4 at 2 weeks after the first immunization and reached 3264 at 3 weeks after the third vaccination (Table 1). The survival rates of the chickens challenged with A/chicken/Yamaguchi/7/2004 (H5N1) are shown in Figure 4. None of the immunized chickens displayed any observable disease after viral challenge. All non-immunized chickens challenged with A/chicken/Yamaguchi/7/2004 (H5N1) died at 2 days p.c. (Figure 4). To assess the potential of the Vlp vaccine to induce immunity and counteract viral shedding, we attempted to retrieve the virus from swabs of the immunized and non-immunized chickens at 2 days p.c. with A/chicken/Yamaguchi/7/2004 (H5N1) (Table 2). The infectivity titers of the recovered viruses from immunized chickens were fundamentally lower than those of non-immunized chickens.

\section{Discussion}

Vlps provide a promising platform as vaccine candidates as they present an antigen in an immunogenic form. One favorable element of the Vlp vaccine approach is that, as a virus-mimicking molecules with various viral antigens and epitopes, Vlps enable a diverse set of protective responses $[27,30]$. Flu Vlp vaccines have been demonstrated to be exceptionally immunogenic and protective in various animal models [27,29-32]. Recently, Prel et al., in addition to Tao et al., presented a Vlp vaccine utilizing a triple baculovirus recombinant that encoded HA, NA, and M1 proteins from H5N3 AIV and demonstrated the protection imparted by these Vlps in Muscovy ducks $[33,34]$. In the present study, we assessed an $\mathrm{H} 5 \mathrm{~N} 1 \mathrm{flu}$ Vlp vaccine containing only three flu virus structural proteins, i.e., HA, NA, and M1, in chickens.

The application of a Vlp vaccine might alleviate the health concerns associated with live-attenuated and inactivated whole virus vaccines. For example, there is evidence that the appearance of the highly pathogenic $\mathrm{H} 5 \mathrm{~N} 2$ of American lineage in Japan resulted from the exchange of the live virus from an incompletely inactivated or contaminated vaccine [35]. In light of the noninfectious nature of Vlps and their lack of viral genomic material, the Vlp vaccine presents an attractive option as a vaccine candidate [31]. Moreover, throughout AIV movement in the poultry population, HA and NA surface antigens undergo systematic amino acid substitutions, which could lead to evasion of the viral resistance conferred by prior vaccination. Because the Vlp vaccine approach permits rapid vaccine preparation, this approach could be utilized to easily redesign the vaccine to reflect transformed or newly arisen strains. The neutralizing reactions afforded after two rounds of immunization were assessed at various time points (weeks 0,4 , and 8 ). At week 8 post-immunization, total IgG was observed in groups that received HA-M1-Vlp and NA-M1 Vlps, and 5-fold higher levels of $\mathrm{H} 5 \mathrm{~N} 1$-specific antibodies were induced in the groups of mice immunized with HA-NA-M1 Vlps (Figure 3A). No significant levels of antibodies specific for A/duck/Hokkaido/vac-1/2004 (H5N1) were detected in groups of mice immunized with HA or HA-NA-negative M1 Vlps. In contrast to HA, the role of NA in vaccine-induced protection has not been investigated, even though both HA and NA are glycoproteins expressed on the surfaces of flu virions. Among the factors that have restricted the investigation of NA-specific vaccines is the trend of immune response towards $\mathrm{HA}$ after flu vaccination. The overwhelming impact of $\mathrm{HA}$ as an antigen was realized when HA and NA were presented in similar viral particles [36,37]. Flu Vlps are a remarkable vehicle for presenting $\mathrm{NA}$ as the major immunogen in the coating of a benign particle. In this time-course study, we explored the effectiveness and immunogenicity of Vlps containing NA. Flu M1 Vlps containing NA were immunogenic and induced functional NA-specific antibodies (Figure 3A). Regardless of the induction of 
Citation: Shimizu M, Yanase S, Myint COO, Okamatsu M, Sakoda Y, et al. (2013) Influenza Virus-like Particles Containing HA, NA, and M1 Induced Protection in Chickens against a Lethal Challenge with the Highly Pathogenic H5N1 Avian Influenza Virus. J Vaccines Vaccin 4: 201. doi: $10.4172 / 2157-7560.1000201$

A

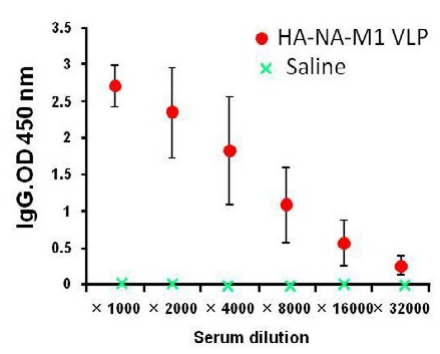

B
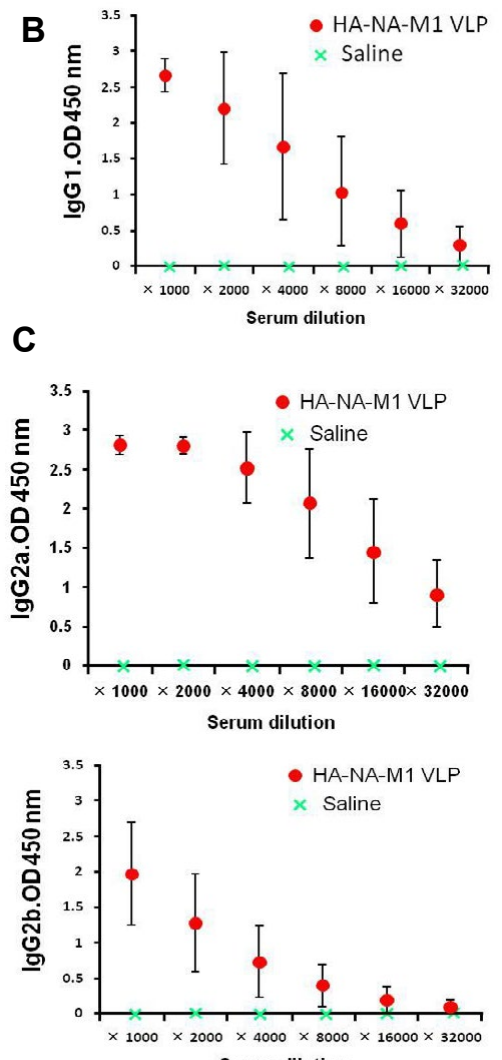
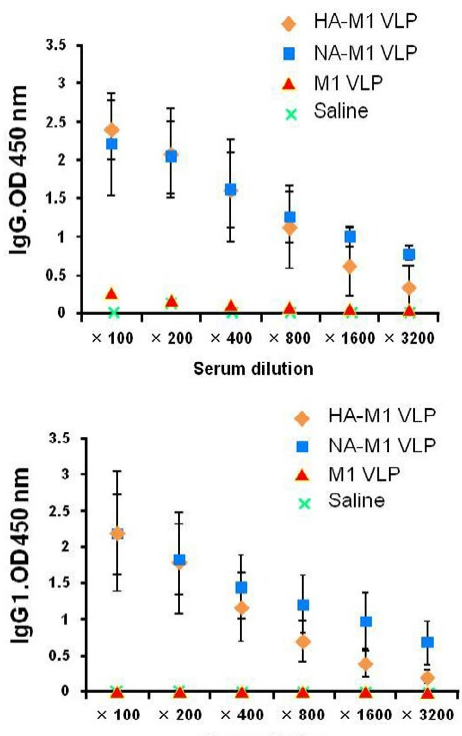

Serum dilution
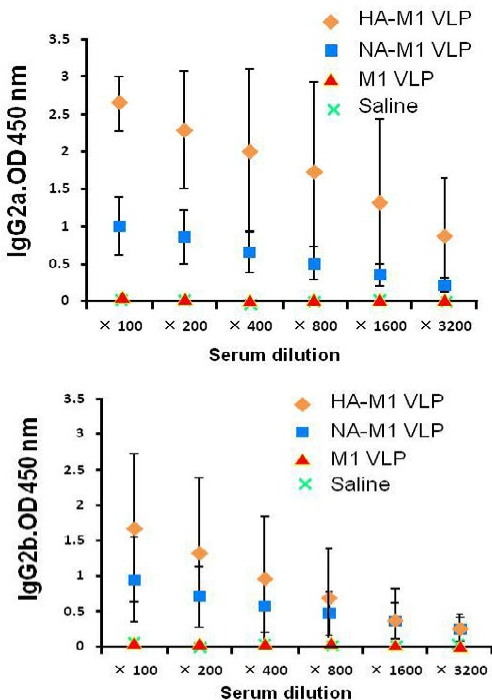

Figure 3: IgG, lgG1, IgG2a and G2b antibody responses. Groups of mice were intramuscularly immunized with HA-NA-M1 VIp at 3 week intervals, and blood samples were collected at various time points (weeks 1 and 4 ) after the final immunization. The sera from individual mice were used, and the average antibody response from each group was determined. Immulon microtiter plates were coated with inactivated influenza H5N1 virus, and mouse antibodies were detected with a horseradish peroxidase-conjugated antibody. The ELISA endpoint titers are expressed as the highest dilutions that yielded optical densities greater than the mean optical density plus 3 standard deviations of similarly diluted negative control sera. (A) lgG antibody reactive to the whole inactivated influenza H5N1 virus. (B) IgG1 antibody reactive to the inactivated influenza H5N1 virus. (C) IgG2a and IgG2b isotype antibodies reactive to the inactivated influenza H5N1 virus.

M1-specific antibodies, the contribution of flu M1 to flu resistance is insignificant $[38,39]$.

We assessed the isotype classes induced by Vlp immunization. The IgG1 antibody, which reflects a Th2-biased response, was discovered to be the predominant subclass in the immunized groups (Figure 3B). In addition, the groups receiving HA-M1 and HA-NA-M1 Vlps predominantly exhibited IgG2a and IgG2b antibodies, indicating Th1-biased responses (Figure 3C). The HA-M1 and HA-NA-M1 Vlps vaccines elicited IgG2a and IgG2b antibodies as well as the IgG1 subclass, characteristic of both Th1 and Th2 immune responses (Figures $3 \mathrm{~B}$ and 3C). Nevertheless, higher levels of H5N1-specific antibodies were induced in the groups of HA-NA-M1 Vlp-immunized mice than HA-M1 Vlp-immunized mice. Furthermore, substantial levels of total IgG and IgG1 isotype antibodies were detected in the NA-M1 Vlp group, whereas only low levels of IgG2a and IgG2b antibodies were detected (Figure 3C).

An analysis of antibody isotypes in immune sera revealed that the immunization of mice (BALB/c) with NA-M1 Vlps predominantly induced IgG1 antibodies. Soluble recombinant HA protein antigens are also known to induce IgG1-dominant antibody responses compared to whole inactivated virus or HA-M1 Vlp antigens, which predominantly induce IgG2a isotype antibodies $[21,22]$. It is probable that NA Vlp without HA, which therefore lacks hemagglutination activity, will be less effective in inducing IgG2a-dominant immune responses than HA Vlp, which has hemagglutination activity.

In the lethal homologous HPAI virus challenge, the group immunized with HA-NA-M1 Vlp demonstrated $100 \%$ protection. In 
Citation: Shimizu M, Yanase S, Myint COO, Okamatsu M, Sakoda Y, et al. (2013) Influenza Virus-like Particles Containing HA, NA, and M1 Induced Protection in Chickens against a Lethal Challenge with the Highly Pathogenic H5N1 Avian Influenza Virus. J Vaccines Vaccin 4: 201. doi: $10.4172 / 2157-7560.1000201$

Page 6 of 7

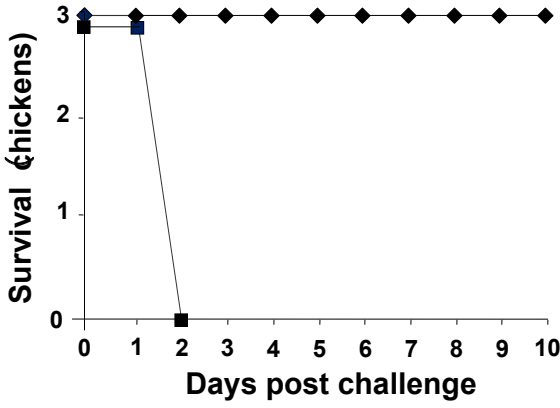

Figure 4: Survival rates of chickens after challenge with A/chicken/ Yamaguchi/7/2004 (H5N1). Four-week-old chickens were immunized intramuscularly three times (at weeks 0,2 , and 4) with $250 \mu \mathrm{gg}$ of $\mathrm{HA}$ NA-M1 VLP formulated in adjuvant. Three weeks later, all of the vaccinated chickens were challenged intranasally with A/chicken/Yamaguchi/7/2004 (H5N1). Symbols: 3 immunized chickens after challenge with A/chicken/ Yamaguchi/7/2004 (H5N1) (black diamond); 3 non-immunized chickens after challenge with A/chicken/Yamaguchi/7/ 2004 (H5N1) (black squares).

\begin{tabular}{|c|c|c|c|c|c|c|}
\hline & \multirow{2}{*}{$\begin{array}{c}\text { Chicken } \\
\text { ID No }\end{array}$} & \multicolumn{5}{|c|}{ Serum HI titers } \\
\cline { 3 - 7 } & & $\mathrm{w} 0$ & $\mathrm{w} 2$ & $\mathrm{w} \mathrm{4}$ & $\mathrm{w} \mathrm{6}$ & $\mathrm{w} 7$ \\
\hline \multirow{2}{*}{$\begin{array}{c}\text { Immunized } \\
\text { Chickens }\end{array}$} & $\# 55$ & $<2$ & 4 & 32 & 64 & 64 \\
\cline { 2 - 7 } & $\# 56$ & $<2$ & 4 & 16 & 64 & 64 \\
\cline { 2 - 7 } & $\# 57$ & $<2$ & 4 & 16 & 32 & 32 \\
\hline \multirow{2}{*}{$\begin{array}{c}\text { Non- } \\
\text { Immunized } \\
\text { Chickens }\end{array}$} & $\# 58$ & $<2$ & $<2$ & $<2$ & $<2$ & $<2$ \\
\cline { 2 - 7 } & $\# 59$ & $<2$ & $<2$ & $<2$ & $<2$ & $<2$ \\
\hline
\end{tabular}

aBlood samples were collected at 0, 2, 4, 6, and 7 weeks after initial inoculation

Table 1: $\mathrm{HI}$ titer of serum samples obtained from vaccinated chickens with HANA-M1 VIp.

\begin{tabular}{|c|c|c|c|c|}
\hline & \multirow{3}{*}{ Chicken ID No } & \multicolumn{3}{|c|}{ Virus recoverya } \\
\hline & & \multirow[t]{2}{*}{ Days p.c. ${ }^{b}$} & \multicolumn{2}{|c|}{$\begin{array}{l}\text { No. of chickens from which } \\
\text { each virus was recovered } \\
\text { Swab (log TCID50/ml) }\end{array}$} \\
\hline & & & Intraoral & Cloacal \\
\hline \multirow{3}{*}{$\begin{array}{l}\text { Immunized } \\
\text { Chickens }\end{array}$} & \#55 & 2 & 0 & 0 \\
\hline & \#56 & 2 & 1.0 & 0 \\
\hline & \#57 & 2 & 0 & 0 \\
\hline \multirow{3}{*}{$\begin{array}{c}\text { Non- } \\
\text { Immunized } \\
\text { Chickens }\end{array}$} & \#58 & $2+$ & 4.3 & 4.0 \\
\hline & \#59 & $2+$ & 5.0 & 4.7 \\
\hline & $\# 60$ & $2+$ & 4.8 & 4.0 \\
\hline
\end{tabular}

a 0 indicates no virus was isolated from chickens

'The number indicates the sampling day p.c.; "+" indicates that chickens died on that day

Table 2: Virus recovery in vaccinated chickens.

contrast, all chickens in the control group died at 2 days p.c. (Figure 4). The challenge virus was detected in several of the intraoral swab samples of chickens immunized with HA-NA-M1 Vlp but not in the cloacal swabs (Table 2). This result indicated that the present vaccine did not completely eliminate the virus, but caused a critical decrease in excessive viral shedding to the chickens, as the administration of an oil-based adjuvanted vaccine has comparable efficacy [40]. Overall, the baculovirus-based Vlp vaccine, HA-NA-M1 Vlp, provided protection to chickens sufficient to prevent observable disease and decreased the amount of viral shedding induced by infection with currently common H5 HPAI viruses.

In conclusion, an intramuscular injection of H5 Vlps provided excellent protection against a lethal viral challenge. The H5 Vlp antibody was more successful in promoting protective Th1-type responses, including IgG2a production. Therefore, flu Vlps could become a significant focus for vaccine strategies, especially if a pandemic occurs. Recognizing the present state of the available vaccines, it is important to carefully examine the relative immunogenicity and protective efficacy of $\mathrm{H} 5$ Vlps in comparison with inactivated whole virus and attenuated live $\mathrm{H} 5 \mathrm{~N} 1$ immunizations.

\section{Conflict of Interest}

The authors do not have any commercial or other associations that might pose a conflict of interest.

\section{Acknowledgment}

We thank Drs. Kawahara, M., Suzuki, T., Suzuki, H., and Nishitsuji, H. for their helpful discussions and excellent work in performing immunizations and ELISAs. This work was supported in part by a grant from the Research and Development Program for New Bio-industry Initiatives from the Ministry of Agriculture, Forestry, and Fisheries of Japan; by a Grant-in-Aid for High Technology Research (no. 09309011) from the Ministry of Education, Science, Sports, and Culture, Japan; and by a grant from the Supporting Program for Creating University Ventures from the Japan Science and Technology Agency.

\section{References}

1. World Health Organization (WHO) (2009) Global Alert and Response. Pandemic (H1N1) 2009.

2. Li YG, Wu JF, Li X (2009) China makes an impressive breakthrough in avian influenza virus research - Discovering the "heart" of avian infl uenza virus. Drug Discov Ther 3: 1

3. WHO. Cumulative number of confirmed cases of avian influenza $\mathrm{A} /(\mathrm{H} 5 \mathrm{~N} 1)$ reported to $\mathrm{WHO}$.

4. Yuen KY, Chan PK, Peiris M, Tsang DN, Que TL, et al. (1998) Clinical features and rapid viral diagnosis of human disease associated with avian influenza $A$ H5N1 virus. Lancet 351: 467-471.

5. Subbarao K, Klimov A, Katz J, Regnery H, Lim W, et al. (1998) Characterization of an avian influenza $A(H 5 N 1)$ virus isolated from a child with a fatal respiratory illness. Science 279: 393-396.

6. Ungchusak K, Auewarakul P, Dowell SF, Kitphati R, Auwanit W, et al. (2005) Probable person-to-person transmission of avian influenza A (H5N1). N Eng J Med 352: 333-340.

7. Kandun IN, Wibisono H, Sedyaningsih ER, Yusharmen, Hadisoedarsuno W, et al. (2006) Three Indonesian clusters of H5N1 virus infection in 2005. N Engl J Med 355: 2186-2194.

8. European Committee for Proprietary Medicinal Products. Guideline on dossier structure and content for pandemic influenza vaccine marketing authorisation application.

9. European Committee for Proprietary Medicinal Products. Guideline on influenza vaccine prepared from viruses with the potential to cause a pandemic and intended for use outside of the core dossier context. European Agency for the Evaluation of Medicinal Products.

10. Food and Drug Administration (FDA) Guidance for Industry. Clinical data needed to support the licensure of pandemic influenza vaccines. US Food and Drug Administration.

11. Leroux-Roels I, Leroux-Roels G (2009) Current status and progress of prepandemic and pandemic influenza vaccine development. Expert Rev Vaccines 8: 401-423

12. Bresson JL, Perronne C, Launay O, Gerdil C, Saville M, et al. (2006) Safety and immunogenicity of an inactivated split-virion influenza A/Vietnam/1194/2004 (H5N1) vaccine: phase I randomised trial. Lancet 367: 1657-1664.

13. Treanor JJ, Campbell JD, Zangwill KM, Rowe T, Wolff M (2006) Safety and immunogenicity of an inactivated subvirion influenza $A(H 5 N 1)$ vaccine. N Engl J Med 354: 1343-1351.

14. Lin J, Zhang J, Dong X, Fang H, Chen J, et al. (2006) Safety and immunogenicity of an inactivated adjuvanted whole-virion influenza $A(H 5 N 1)$ vaccine: a phase I randomised controlled trial. Lancet 368: 991-997. 
Citation: Shimizu M, Yanase S, Myint COO, Okamatsu M, Sakoda Y, et al. (2013) Influenza Virus-like Particles Containing HA, NA, and M1 Induced Protection in Chickens against a Lethal Challenge with the Highly Pathogenic H5N1 Avian Influenza Virus. J Vaccines Vaccin 4: 201. doi: $10.4172 / 2157-7560.1000201$

15. Leroux-Roels I, Borkowski A, Vanwolleghem T, Dramé M, Clement F, et al. (2007) Antigen sparing and cross-reactive immunity with an adjuvanted rH5N1 prototype pandemic influenza vaccine: a randomised controlled trial. Lance 370: 580-589.

16. Galarza JM, Latham T, Cupo A (2005) Virus-like particle (VLP) vaccine conferred complete protection against a lethal influenza virus challenge. Viral Immunol 18: 244-251.

17. Pushko P, Tumpey TM, Bu F, Knell J, Robinson R, et al. (2005) Influenza viruslike particles comprised of the HA, NA, and M1 proteins of H9N2 influenza virus induce protective immune responses in BALB/c mice. Vaccine 23: 5751-5759.

18. Pushko P, Tumpey TM, Van Hoeven N, Belser JA, Robinson R, et al. (2007) Evaluation of influenza virus-like particles and Novasome adjuvant as candidate vaccine for avian influenza. Vaccine 25: 4283-4290.

19. Quan FS, Huang C, Compans RW, Kang SM (2007) Virus-like particle vaccine induces protective immunity against homologous and heterologous strains of influenza virus. J Virol 81: 3514-3524.

20. Bright RA, Carter DM, Daniluk S, Toapanta FR, Ahmad A, et al. (2007) Influenza virus-like particles elicit broader immune responses than whole virion inactivated influenza virus or recombinant hemagglutinin. Vaccine 25: 3871-3878.

21. Bright RA, Carter DM, Crevar CJ, Toapanta FR, Steckbeck JD, et al. (2008) Cross-clade protective immune responses to influenza viruses with $\mathrm{H} 5 \mathrm{~N} 1 \mathrm{HA}$ and NA elicited by an influenza virus-like particle. PLoS One 3: e1501.

22. Matassov D, Cupo A, Galarza JM (2007) A novel intranasal virus-like particle (VLP) vaccine designed to protect against the pandemic 1918 influenza A virus (H1N1). Viral Immunol 20: 441-452.

23. Quan FS, Steinhauer D, Huang C, Ross TM, Compans RW, et al. (2008) A bivalent influenza VLP vaccine confers complete inhibition of virus replication in lungs. Vaccine 26: 3352-3361.

24. Abe T, Takahashi $\mathrm{H}$, Hamazaki $\mathrm{H}$, Miyano-Kurosaki $\mathrm{N}$, Matsuura $\mathrm{Y}$, et al. (2003) Baculovirus induces an innate immune response and confers protection from lethal influenza virus infection in mice. J Immunol 171: 1133-1139.

25. Soda K, Sakoda Y, Isoda N, Kajihara M, Haraguchi Y, et al. (2008) Development of vaccine strains of $\mathrm{H} 5$ and $\mathrm{H} 7$ influenza viruses. Jpn J Vet Res 55: 93-98.

26. Donald HB, Isaacs A (1954) Counts of influenza virus particles. J Gen Microbio 10: $457-464$

27. Anonymous. Identification of influenza isolates by hemagglutination inhibition (2002) Geneva, World Health Organization 28-36.

28. Mase M, Eto M, Tanimura N, Imai K, Tsukamoto K, et al. (2005) Isolation of a genotypically unique $\mathrm{H} 5 \mathrm{~N} 1$ influenza virus from duck meat imported into Japan from China. Virology 339: 101-109.

29. Quan FS, Kim MC, Lee BJ, Song JM, Compans RW, et al. (2012) Influenza M1 VLPs containing neuraminidase induce heterosubtypic cross-protection. Virology 430: 127-135

30. Ross TM, Mahmood K, Crevar CJ, Schneider-Ohrum K, Heaton PM, et al. (2009) A trivalent virus-like particle vaccine elicits protective immune responses against seasonal influenza strains in mice and ferrets. PLoS One 4: e6032.

31. Kang SM, Pushko P, Bright RA, Smith G, Compans RW (2009) Influenza viruslike particles as pandemic vaccines. Curr Top Microbiol Immunol 333: 269-289.

32. Song JM, Hossain J, Yoo DG, Lipatov AS, Davis CT, et al. (2010) Protective immunity against $\mathrm{H} 5 \mathrm{~N} 1$ influenza virus by a single dose vaccination with viruslike particles. Virology 405: 165-175.

33. Prel A, Le Gall-Reculé G, Jestin V (2008) Achievement of avian influenza viruslike particles that could be used as a subunit vaccine against low-pathogenic avian influenza strains in ducks. Avian Pathol 37: 513-520.

34. Tao P, Luo M, Zhu D, Qu S, Yang Z, et al. (2009) Virus-like particle vaccine comprised of the HA, NA, and M1 proteins of an avian isolated H5N1 influenza virus induces protective immunity against homologous and heterologous strains in mice. Viral Immunol 22: 273-281.

35. Krauss S, Obert CA, Franks J, Walker D, Jones K, et al. (2007) Influenza in migratory birds and evidence of limited intercontinental virus exchange. PLoS Pathog 3: e167.

36. Johansson BE, Moran TM, Kilbourne ED (1987) Antigen-presenting B cells and helper $T$ cells cooperatively mediate intravirionic antigenic competition between influenza A virus surface glycoproteins. Proc Natl Acad Sci USA 84: 6869-6873.

37. Johansson BE, Kilbourne ED (1993) Dissociation of influenza virus hemagglutinin and neuraminidase eliminates their intravirionic antigenic competition. J Virol 67: 5721-5723.

38. Chen Z, Sahashi Y, Matsuo K, Asanuma H, Takahashi H, et al. (1998) Comparison of the ability of viral protein-expressing plasmid DNAs to protect against influenza. Vaccine 16: 1544-1549.

39. Wiesener N, Schütze T, Lapp S, Lehmann M, Jarasch-Althof N, et al. (2011) Analysis of different DNA vaccines for protection of experimental influenza $A$ virus infection. Viral Immunol 24: 321-330.

40. Isoda N, Sakoda Y, Kishida N, Soda K, Sakabe S, et al. (2008) Potency of an inactivated avian influenza vaccine prepared from a non-pathogenic H5N1 reassortant virus generated between isolates from migratory ducks in Asia. Arch Virol 153: 1685-1692. 